\title{
Implicitly and Densely Discrete Black-Box Optimization Problems*
}

\author{
L. N. Vicente ${ }^{\dagger}$
}

February 26, 2009

\begin{abstract}
This paper addresses derivative-free optimization problems where the variables lie implicitly in an unknown discrete closed set. The evaluation of the objective function follows a projection onto the discrete set, which is assumed dense (and not sparse as in integer programming). Such a mathematical setting is a rough representation of what is common in many real-life applications where, despite the continuous nature of the underlying models, a number of practical issues dictate rounding of values or projection to nearby feasible figures.

We discuss a definition of minimization for these implicitly discrete problems and outline a direct-search algorithm framework for its solution. The main asymptotic properties of the algorithm are analyzed and numerically illustrated.
\end{abstract}

Keywords: Derivative-free optimization, (dense) discrete optimization, direct search, projection, rounding, location, grids.

\section{The implicitly and densely discrete problem}

Many optimization problems are only apparently continuous. The practical nature of many applications involves an underlying discrete (usually unknown) structure which is not taken explicitly in the modeling or solution phases and only revealed later when the result determined by some optimization process is actually applied. In other application problems, which are of interest to us in this paper, an evaluation of the objective function is done first by 'rounding' the values of the variables to allowable figures or by 'projecting' them to nearby values or grid points where it is possible or desirable to evaluate the real function. The underlying discrete structure is thus unknown to the optimizer and its manifestation is detected only when a function evaluation is demanded.

\footnotetext{
*Support has been provided by FCT under grants POCI/MAT/59442/2004 and PTDC/MAT/64838/2006.

${ }^{\dagger}$ CMUC, Department of Mathematics, University of Coimbra, 3001-454 Coimbra, Portugal (lnv@mat.uc.pt).
} 
We can pose the problem under consideration as follows

$$
\begin{array}{ll}
\min _{x \in \mathcal{L}} & f(x) \\
\text { s.t. } & x \in \Omega,
\end{array}
$$

where $\Omega \subseteq \mathbb{R}^{n}$ is some feasible region and $\mathcal{L}$ is an unknown set in $\mathbb{R}^{n}$ that meets the following requirements:

(L1) The set is discrete (i.e., for every $x \in \mathcal{L}$ there exists a neighborhood $\mathcal{N}_{x} \subseteq \mathbb{R}^{n}$ of $x$ such that $\left.\mathcal{L} \cap \mathcal{N}_{x}=\{x\}\right)$.

(L2) The set is closed.

(L3) The distance between points in $\mathbb{R}^{n}$ and the closest points in $\mathcal{L}$ cannot be arbitrarily large.

Due to (L1) and (L2), $\mathcal{L}$ has no any accumulation points or, less formally, points in $\mathcal{L}$ are not arbitrarily close to each other. Also, because of (L1) and (L2) the intersection of $\mathcal{L}$ with a compact set must necessarily be finite. Integer lattices are examples of sets satisfying conditions (L1)-(L3). Let $P_{\mathcal{L}}: 2^{\mathbb{R}^{n}} \rightarrow 2^{\mathcal{L}}$ denote a (idempotent) projection operator onto $\mathcal{L}$.

Definition 1.1 We say that $x_{*} \in \Omega \cap \mathcal{L}$ is an implicitly and densely discrete local minimizer if for some $\sigma_{\text {out }}>\sigma_{\text {in }}>0$, the following conditions are satisfied:

$$
\begin{aligned}
& f\left(x_{*}\right) \leq f(x) \quad \forall x \in R_{\text {out }}, \\
& f\left(x_{*}\right) \leq f(x) \quad \forall x \in R_{\text {between }}, \\
& P_{\mathcal{L}}\left(\left\{y \in \mathbb{R}^{n}:\left\|y-x_{*}\right\| \leq \sigma_{\text {in }}\right\} \cap \Omega\right)=\left\{x_{*}\right\},
\end{aligned}
$$

where

$$
\begin{aligned}
R_{\text {out }} & =P_{\mathcal{L}}\left(\left\{y \in \mathbb{R}^{n}:\left\|y-x_{*}\right\|=\sigma_{\text {out }}\right\}\right) \cap \Omega, \\
R_{\text {between }} & =\left[(\Omega \cap \mathcal{L}) \cap\left\{y \in \mathbb{R}^{n}: \sigma_{\text {in }}<\left\|y-x_{*}\right\|<\sigma_{\text {out }}\right\}\right] \backslash R_{\text {out }} .
\end{aligned}
$$

We depict an example of the sets $R_{\text {out }}$ and $R_{\text {between }}$ in Figure 1.

Definition 1.1 implies a natural notion of local minimization (when $P_{\mathcal{L}}$ is the minimum $\ell_{2}$-distance projection operator), in the sense that if $x_{*} \in \Omega \cap \mathcal{L}$ is an implicitly and densely discrete local minimizer there is a neighborhood $\mathcal{N}_{x_{*}}$ of $x_{*}$ such that $f\left(x_{*}\right) \leq f(x)$ for all $x \in \Omega \cap \mathcal{L} \cap \mathcal{N}_{x_{*}}$ and $\Omega \cap \mathcal{L} \cap \mathcal{N}_{x_{*}} \neq\left\{x_{*}\right\}$.

Note also that the existence of a $\sigma_{i n}>0$ such that condition (4) is satisfied is trivially guaranteed given the properties of $\mathcal{L}$. However, our definition splits the main properties of local minimization into (2) and (3) and thus introduces $R_{\text {between }}$, which in turn depends on $\sigma_{i n}$. 
These definitions of local minimization might not be appropriate for sparsely discrete problems (such as integer programming problems). According to our definition, for example, all the points in $\mathcal{L} \cap \Omega=\{-13,-8,-4,-1,1,4,8,13\}$ are local minimizers for $f(x)=-|x|$ when $P_{\mathcal{L}}$ is the minimum $\ell_{2}$-distance projection operator. This example was pointed out to us by Audet [2] who suggested an alternative definition which does not break down for this example. However, in this paper we have in mind densely discrete optimization problems for which Definition 1.1 seems appropriate. Also, as we will see in this paper, Definition 1.1 suits the convergence purposes of a vast class of direct-search methods.

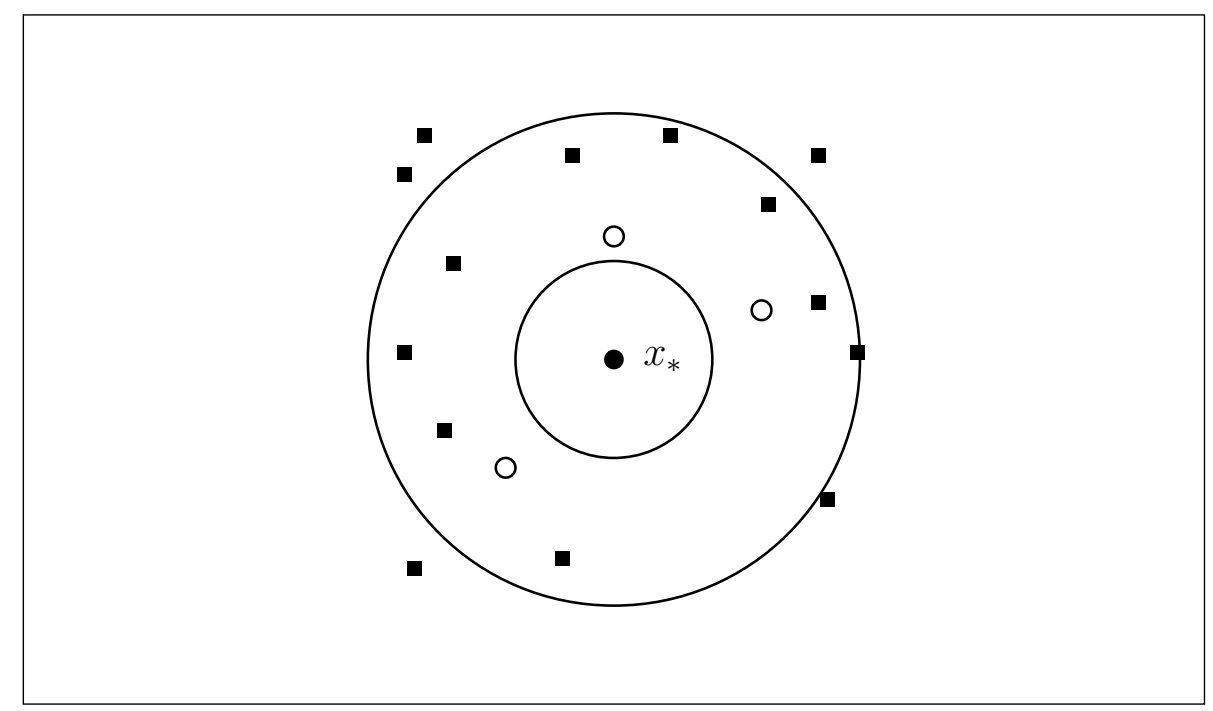

Figure 1: Example where the filled squares correspond to points in $R_{\text {out }}$ and the empty circles to points in $R_{\text {between }}$. The circles have radii $\sigma_{\text {in }}$ (inner) and $\sigma_{\text {out }}$ (outer).

In an algorithmic context we assume that the projection operator always returns a singleton when operating on a single point, and any ties are broken according to some application-dependent criterion. In such situations, $P_{\mathcal{L}}(x)=y$ corresponds to $P_{\mathcal{L}}(\{x\})=$ $\{y\}$. For simplicity, we will consider that $P_{\mathcal{L}}$ is the minimum $\ell_{2}$-distance projection operator, but this can be relaxed. In fact, we will see that all is needed is that, whenever the distance between a point in $\mathbb{R}^{n}$ and its projection become arbitrarily large, Condition (L3) is violated. 


\section{A direct-search approach}

Below we describe a general direct-search method for the solution of the implicitly and densely discrete optimization problem (1). We make use of the extreme barrier function:

$$
f_{\Omega}(x)= \begin{cases}f(x) & \text { if } x \in \Omega \\ +\infty & \text { otherwise }\end{cases}
$$

The set of directions $D_{k}$ used in the algorithm is not necessarily a positive spanning set.

The discrete aspect is detected implicitly each time a function evaluation is attempted. The function is calculated at $P_{\mathcal{L}}\left(x_{k}+\alpha_{k} d\right)$ and not at $x_{k}+\alpha_{k} d$, where $x_{k}$ is the current point, $d$ a direction, and $\alpha_{k}$ the mesh or step size parameter. The user returns to the optimizer the point $P_{\mathcal{L}}\left(x_{k}+\alpha_{k} d\right)$ and the objective value $f\left(P_{\mathcal{L}}\left(x_{k}+\alpha_{k} d\right)\right)$ if $P_{\mathcal{L}}\left(x_{k}+\alpha_{k} d\right) \in \Omega$. Note that this might be made in two separate stages (projection and evaluation) depending on the application. If it is done in two stages, then the algorithm takes advantage of it. Note that if the user only returns the function value, then the corresponding class of problems is outside the scope of this paper. In such cases, we would be dealing with 'noisy' problems or problems of 'dynamic accuracy' (this latter term is the one used in the book [4]), which are treated differently.

\section{Algorithm 2.1 (Direct-search algorithm)}

Step 0 (initialization): Let $x_{0} \in \Omega \cap \mathcal{L}$ be provided by the user and select $\alpha_{0}>0$ and $c \in(0,1)$.

Step 1 (polling): Select a set of directions $D_{k}$.

For all $d \in D_{k}$ :

(A) Ask the user to provide $P_{\mathcal{L}}\left(x_{k}+\alpha_{k} d\right)$.

(B) If $\left\|P_{\mathcal{L}}\left(x_{k}+\alpha_{k} d\right)-x_{k}\right\| \leq c \alpha_{k}$ then stop polling, set $x_{k+1}=x_{k}$ (and, for later presentation, set $v_{k}=d$ ), increase $\alpha_{k}$, increment $k$ by one unit, and return to the beginning of Step 1.

(C) Ask the user to provide $f_{\Omega}\left(P_{\mathcal{L}}\left(x_{k}+\alpha_{k} d\right)\right.$ ) (if not yet available when computing $P_{\mathcal{L}}\left(x_{k}+\alpha_{k} d\right)$ in (A) above).

(D) Otherwise $\left(\left\|P_{\mathcal{L}}\left(x_{k}+\alpha_{k} d\right)-x_{k}\right\|>c \alpha_{k}\right)$ then

- If $f_{\Omega}\left(P_{\mathcal{L}}\left(x_{k}+\alpha_{k} d\right)\right)<f\left(x_{k}\right)$ then stop polling, set $x_{k+1}=P_{\mathcal{L}}\left(x_{k}+\alpha_{k} d\right)$ (and, for later presentation, set $d_{k}=d$ ), increase or retain $\alpha_{k}$, increase $k$ by one unit, and return to the beginning of Step 1.

- Otherwise continue the polling loop.

Step 2 (unsuccessful polling): When $f_{\Omega}\left(P_{\mathcal{L}}\left(x_{k}+\alpha_{k} d\right)\right) \geq f\left(x_{k}\right)$ and $\| P_{\mathcal{L}}\left(x_{k}+\alpha_{k} d\right)-$ $x_{k} \|>c \alpha_{k}$ for all $d \in D_{k}$, set $x_{k+1}=x_{k}$, decrease $\alpha_{k}$, increment $k$ by one unit, and return to the beginning of Step 1. 
Note that the sequence of iterates $\left\{x_{k}\right\}$ generated by this algorithm necessarily lies in $\Omega \cap \mathcal{L}$.

We ask the sets of directions $D_{k}$ to satisfy the following assumptions:

(D1) $\|d\| \geq 1 \forall d \in D_{k}, \forall k$.

(D2) $\alpha_{k} d \rightarrow 0 \forall d \in D_{k}$ whenever $\alpha_{k} \rightarrow 0$ for some subsequence.

(D3) $\bigcup_{k \in K} D_{k}$ is dense in the unit sphere of $\mathbb{R}^{n}$ for all subsequences $K$ for which $\alpha_{k} \rightarrow \alpha>0$.

These conditions, in particular (D2), are compatible with those imposed on positive spanning sets $D_{k}$ by mesh adaptive direct search (MADS) methods [3] for continuous problems.

Also, one can see that the only difference between the standard polling procedure of directional direct-search methods and the polling scheme of Algorithm 2.1 is the introduction of the test $\left\|P_{\mathcal{L}}\left(x_{k}+\alpha_{k} d\right)-x_{k}\right\| \leq c \alpha_{k}$. Thus, one could use this test in the polling procedure of directional direct-search methods and, based on it, one could detect whether the algorithmic mesh associated with an iteration of these methods is too fine when compared to $\mathcal{L}$.

\section{Analysis of the direct-search method}

Now we analyze the convergence properties of Algorithm 2.1. Essentially we will show that part of the conditions of implicitly and densely discrete local minimization are asymptotically satisfied.

Theorem 3.1 Let $\left\{x_{k}\right\}$ be a sequence of iterates generated by Algorithm 2.1 where the sets of polling directions satisfy Assumptions (D1)-(D3).

If $L\left(x_{0}\right)=\left\{x \in \mathbb{R}^{n}: f(x) \leq f\left(x_{0}\right)\right\}$ is bounded then there exist $x_{*} \in \Omega \cap \mathcal{L}$ and subsequences $K_{\text {in }}$ and $K_{\text {out }}$ and positive numbers $0<\alpha_{\text {in }}<\alpha_{\text {out }}$ such that

(i) $\alpha_{k} \rightarrow \alpha_{\text {out }}$ when $k \in K_{\text {out }}$ and

$$
f_{\Omega}\left(P_{\mathcal{L}}\left(x_{*}+\alpha_{k} d\right)\right) \geq f\left(x_{*}\right) \quad \forall d \in D_{k}, \forall k \in K_{\text {out }},
$$

where $\cup_{k \in K_{\text {out }}} D_{k}$ is dense in the unit sphere in $\mathbb{R}^{n}$, and

(ii) $\alpha_{k} \rightarrow \alpha_{\text {in }}$ when $k \in K_{\text {in }}$ and

$$
\exists_{v_{k} \in D_{k},\left\|v_{k}\right\| \geq 1}:\left\|P_{\mathcal{L}}\left(x_{*}+\alpha_{k} v_{k}\right)-x_{*}\right\| \leq c \alpha_{i n} \quad \forall k \in K_{\text {in }},
$$

with $\left\|v_{k}\right\| \rightarrow 1$ in $K_{\text {in }}$. 
Proof. First we note that, because $L\left(x_{0}\right)$ is bounded, the set $\mathcal{L} \cap L\left(x_{0}\right)$ is finite. Since the algorithm only moves to a new point in $\mathcal{L}$ when a decrease is found, there must exist a $\bar{k}$ such that

$$
x_{k}=x_{\bar{k}}=x_{*} \quad \forall k \geq \bar{k} .
$$

Let us first prove that $\left\{\alpha_{k}\right\}$ is a bounded sequence. For this purpose let us assume that there exists a subsequence driving $\alpha_{k}$ to $+\infty$. If that is the case, there must exist another subsequence denoted by $K$ where $\alpha_{k}$ is increased and $\alpha_{k} \rightarrow+\infty$ for $k \in K$. From the algorithm we have $\left\|P_{\mathcal{L}}\left(x_{*}+\alpha_{k} v_{k}\right)-x_{*}\right\| \leq c \alpha_{k}$ for $k$ sufficiently large in $K$, and it can be easily proved for such values of $k$ that

$$
\left\|\left(x_{*}+\alpha_{k} v_{k}\right)-P_{\mathcal{L}}\left(x_{*}+\alpha_{k} v_{k}\right)\right\| \geq\left(\left\|v_{k}\right\|-c\right) \alpha_{k} .
$$

Using Assumption (D1) and taking $\alpha_{k} \rightarrow+\infty$ for $k \in K$ in both sides of this inequality contradict Condition (L3) of the definition of $\mathcal{L}$ (note that it is here that one needs to qualify the projection operator).

It can also be proved that $\alpha_{k}$ is uniformly bounded away from zero. In fact, if there was a subsequence driving $\alpha_{k}$ to zero, then there would exist another subsequence, denoted by $J$, for which $\alpha_{k}$ is decreased (which then means that $\left\|P_{\mathcal{L}}\left(x_{*}+\alpha_{k} d\right)-x_{*}\right\|>c \alpha_{k}>0$ for $k$ sufficiently large) and $\alpha_{k} \rightarrow 0$ for $k \in J$. From Assumption (D2) we obtain $\alpha_{k} d \rightarrow 0$ in $J$ for some $d \in D_{k}$. Thus, we derive that

$$
x_{*}+\alpha_{k} d \rightarrow x_{*} \in \mathcal{L} \quad \text { and } \quad P_{\mathcal{L}}\left(x_{*}+\alpha_{k} d\right) \neq x_{*} \forall k \in J \text { sufficiently large, }
$$

which contradicts conditions (L1)-(L2) of the definition of $\mathcal{L}$.

Since $\alpha_{k}$ is uniformly bounded from above and away from zero, there must exist subsequences $K_{\text {in }}$ and $K_{\text {out }}$ and positive numbers $0<\alpha_{\text {in }}<\alpha_{\text {out }}$ such that (i) $\alpha_{k} \rightarrow \alpha_{\text {out }}$ and $\alpha_{k}$ is decreased for all $k \in K_{\text {out }}$ and (ii) $\alpha_{k} \rightarrow \alpha_{\text {in }}$ and $\alpha_{k}$ is increased for all $k \in K_{\text {in }}$. Thus,

$$
f_{\Omega}\left(P_{\mathcal{L}}\left(x_{*}+\alpha_{k} d\right)\right) \geq f\left(x_{*}\right) \quad \forall d \in D_{k}, \forall k \in K_{\text {out }},
$$

and

$$
\left\|P_{\mathcal{L}}\left(x_{*}+\alpha_{k} v_{k}\right)-x_{*}\right\| \leq c \alpha_{k} \quad \text { for some } v_{k} \in D_{k}, \forall k \in K_{i n} .
$$

The proof is completed by using Assumptions (D1) and (D3).

It is clear that the point $x_{*}$ identified in this theorem satisfies a condition (see $(7)$ ) which is practically the same as (2), with $\sigma_{\text {out }}=\alpha_{\text {out }}$.

Condition (4) is roughly approximated by (8) if the constant $c$ is small and $\left\{v_{k}: k \in\right.$ $\left.K_{i n}\right\}$ is dense in the unit sphere. The absolute satisfaction of (4) would require some form of dense sampling close to $x_{*}$.

What is clearly missing in the result of Theorem 3.1 is a condition of type (3). However, to capture the points in $R_{\text {between }}$ seems a rather difficult task for any reasonable algorithmic framework, since it seems to require more than the generation of directions dense in the unit sphere (which is what is required in this paper and in MADS [3]), i.e., something like dense sampling in the unit ball seems to be also necessary for such a purpose. 


\section{A numerical illustration}

We made some experiments in MATLAB [1] to observe and possibly confirm our theoretical findings. The implementation chosen for Algorithm 2.1 was rather simple. The set $D_{k}$ is set to the positive basis $\left[Q_{k}-Q_{k}\right]$ where $Q_{k}$ is an orthogonal matrix computed by first randomly generating the first column (independently of $\alpha_{k}$ ) and then computing the remaining columns using a QR factorization. We report here some results obtained for the minimization in $\mathcal{L}$ of a quadratic function perturbed by oscillatory noise:

$$
\begin{array}{rl}
\min _{\left(x_{1}, x_{2}\right) \in \mathcal{L}} & 10\left(x_{1}^{2}\right)\left(1+0.75 \cos \left(70 x_{1}\right) / 12\right)+\cos \left(100 x_{1}\right)^{2} / 24+ \\
& 2\left(x_{2}^{2}\right)\left(1+0.75 \cos \left(70 x_{2}\right) / 12\right)+\cos \left(100 x_{2}\right)^{2} / 24+4 x_{1} x_{2} .
\end{array}
$$

The unique minimizer of this problem is $x_{*}=(0,0)$. The set $\mathcal{L}$ has been chosen as the integer lattice $\left\{\gamma z: z \in \mathbb{Z}^{n}\right\}$, with $\gamma \neq 0$. For these experiments we ran the algorithm for a specified number of iterations (500 in the first case and 1000 in the second one). Introducing an appropriate stopping criterion for the algorithm would require some practical rules to approximate the inferior and superior limits of the sequence $\left\{\alpha_{k}\right\}$. In Figure 2, we plot the behavior of the mesh or step size parameter $\alpha_{k}$. One can easily observe in both plots that $\alpha_{k}$ oscillates between an upper and a lower value as predicted by Theorem 3.1.

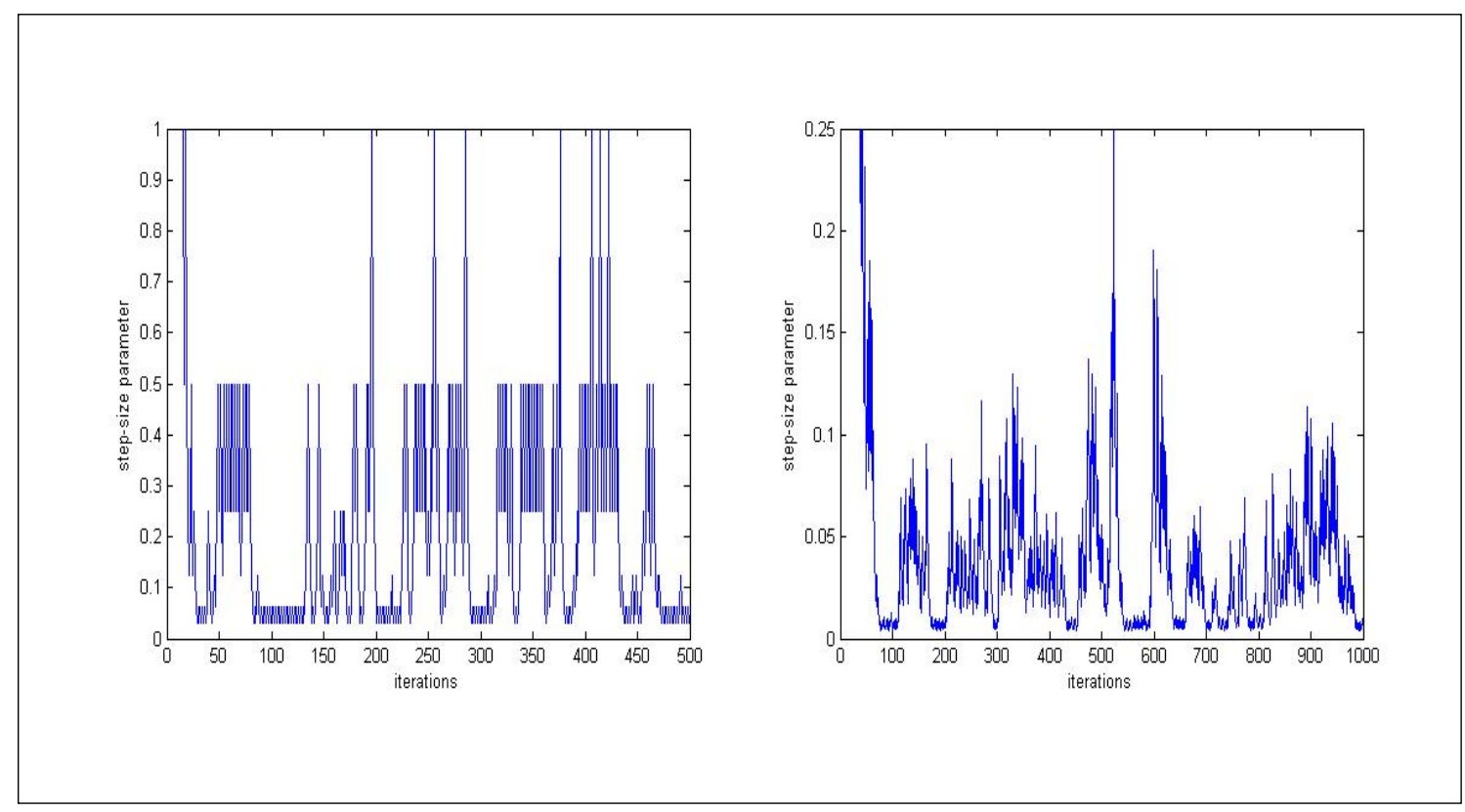

Figure 2: Illustration of the behavior of the mesh or step size parameter $\alpha_{k}$ in Algorithm 2.1. For the run reported on the left we chose $\gamma=0.1$ and $c=0.95$, increased $\alpha_{k}$ by a factor of 2 (in both occurrences of the algorithm), and decreased it by a factor of 0.5 in unsuccessful polling steps. The plot on the right corresponds to a finer lattice $(\gamma=0.01)$ and a decreasing factor of 0.75 . Both runs started from $x_{0}=(5,-10)$ and $\alpha_{0}=1$. 


\section{$5 \quad$ Discussion and open issues}

This paper is a first attempt to shed some light on the solution of implicitly discrete black-box optimization problems. These problems are characterized by the existence of an implicit and unknown discrete set (assumed relatively dense) where optimization points are first projected before the objective function is evaluated. In this paper we suggested a reasonable working definition of local minimization which suits the needs of direct-search methods.

The proposed direct-search method is directional and resembles MADS [3] when ' $\mathcal{L} \rightarrow$ $\mathbb{R}^{n}$. The denser the set $\mathcal{L}$ is in $\mathbb{R}^{n}$ the smaller the value of $\sigma_{i n}$ becomes (see Definition 1.1). When one suspects that $\mathcal{L}$ is 'sufficiently dense' in $\mathbb{R}^{n}$, the set of directions must also satisfy the property that for all subsequences driving $\alpha_{k}$ to zero the union of the $D_{k}$ 's is dense in $\mathbb{R}^{n}$. It is also important to remark that the suggested polling procedure is only slightly different from the traditional (see (B) in Step 1 of Algorithm 2.1) which makes it applicable to most directional direct-search methods.

A number of issues are of interest and have not been fully addressed here. The following is an attempt to enumerate some of them:

- Can we incorporate other mechanisms in the suggested direct-search framework to look for points in $R_{\text {between }}$ ? Could we, for instance, target different circles instead of one (the one now corresponding to $\sigma_{\text {out }}$ in Definition 1.1)? We doubt, however, that it would be possible to capture all the points in $R_{\text {between }}$ without some form of dense circles in the unit ball.

- Would it be possible to develop stopping criteria which can be satisfied asymptotically without approximating the inferior and superior limits of the sequence $\left\{\alpha_{k}\right\}$ ?

- It is known that pattern search algorithms [5, 6] generate points in an integer lattice. Would it be possible for such type of direct-search algorithms to 'align' their integer lattices with the application discrete structure? Under what conditions would that be achievable?

- We wanted the current approach to be as general as possible. We have not taken advantage, for instance, of the size of $\left\|P_{\mathcal{L}}\left(x_{k}+\alpha_{k} d\right)-\left(x_{k}+\alpha_{k} d\right)\right\|$ in the algorithm when compared to $\alpha_{k}$.

- Is it possible to derive other definitions of local minimization for implicitly and densely discrete problems both capable of giving a satisfactory answer for sparsely discrete problems and of fitting the convergence needs of direct-search methods?

\section{Acknowledgement}

The author would like to thank two anonymous referees for their comments. One of the referees, in particular, raised an interesting application for implicitly and densely discrete 
black-box optimization (the querying of a cache of previously evaluated points in derivativefree optimization): Typically, a computational savings is achieved by first searching through the cache before evaluating a potentially expensive objective function at a trial point. If the trial point is "sufficiently close" to the point in the cache, then the function returns the function value of the cached point instead of evaluating the trial point at high cost.

\section{References}

[1] MATLAB, The MathWorks Inc. http://www.mathworks.com.

[2] C. Audet, Private communication.

[3] C. Audet And J. E. Dennis JR., Mesh adaptive direct search algorithms for constrained optimization, SIAM J. Optim., 17 (2006), pp. 188-217.

[4] A. R. Conn, N. I. M. Gould, And P. L. Toint, Trust-Region Methods, MPS-SIAM Series on Optimization, SIAM, Philadelphia, 2000.

[5] A. R. Conn, K. Scheinberg, And L. N. Vicente, Introduction to Derivative-Free Optimization, MPS-SIAM Series on Optimization, SIAM, Philadelphia, 2009.

[6] T. G. Kolda, R. M. Lewis, And V. Torczon, Optimization by direct search: New perspectives on some classical and modern methods, SIAM Rev., 45 (2003), pp. 385-482. 